\title{
Perinephric Hematoma Following Renal Biopsy: A Case Report and Literature Review
}

\section{Girish P Vakrani ${ }^{1 *}$, Nambakam Tanuja Subramanyam² and Hareesh Babu ${ }^{1}$}

${ }^{1}$ Department of Nephrology, Affiliated to Vydehi Institute of Medical Sciences and Research Center, Bangalore, India

${ }^{2}$ Department of General Medicine, Currently Affiliated to Vydehi Institute of Medical Sciences and Research Center, Bangalore, India

\begin{abstract}
Background: The risks associated with performing a percutaneous renal biopsy have substantially decreased in the past two decades because of technical advances in the method. However, bleeding complications still occur, resulting in increased hospital stay, treatment costs and even mortality.
\end{abstract}

Objective: The purpose of this case reporting is to discuss about life threatening complication like perinephric hematoma following a renal biopsy in a high risk patient with severe renal failure.

Methods: The clinical, radiological findings, prognosis of a massive perinephric hematoma following a renal biopsy in a high risk patient with severe renal failure are discussed.

Results: We report a case of 39 year old, male, non-diabetic, hypertensive since 5 years who presented with unexplained severe renal failure. After dialyzing adequately, he was subjected to right renal biopsy using real time ultrasound guided automated renal biopsy gun. Post biopsy was uneventful, until 72 hours when he developed massive right perinephric hematoma following a heparin-free hemodialysis. He continued to be hypotensive despite hemostatics, blood products, and plasma expander transfusion. Unfortunately, he expired before definitive management like renal angiogram and intervention was attempted.

Conclusions: To the best of our knowledge, this is one of few reports of the occurrence of life threatening complication like perinephric hematoma in a patient with severe renal failure on heparin free hemodialysis 72 hours following a renal biopsy.

Keywords: Perinephric hematoma; Real time ultrasound guided renal biopsy gun; Severe renal failure

\section{Introduction}

The risks associated with performing a percutaneous renal biopsy have substantially decreased in the past two decades because of technical advances in the method. However, bleeding complications still occur, resulting in increased hospital stay, treatment costs and even mortality.

\section{Case Report}

A case of 39 year old, male, non diabetic, hypertensive since 5 years on medications regularly presented with swelling of legs, decreased urine since 1 week duration. Physical examination revealed blood pressure of $140 / 94 \mathrm{mmHg}$, a pulse rate of 72 beats/minute, and pitting pedal edema. Systemic examination was normal.

Investigations showed sub-nephrotic proteinuria, microscopic hematuria, hemoglobin was $9 \mathrm{~g} / \mathrm{dl}$ (normal range $12-15 \mathrm{~g} / \mathrm{dL}$ ), serum creatinine was $6.56 \mathrm{mg} / \mathrm{dl}$ (normal 0.6-1.5 mg/dL), blood urea was 114 $\mathrm{mg} / \mathrm{dl}$ (normal $15-40 \mathrm{mg} / \mathrm{dL}$ ), serum albumin was $2.3 \mathrm{~g} / \mathrm{dl}$ (normal 3.0$5.0 \mathrm{~g} / \mathrm{dL}$ ), viral serology (HIV , HCV , HBsAg) were negative, ultrasound abdomen showed grade 1 nephropathy (right kidney $89 \mathrm{~mm}$, left kidney $89 \mathrm{~mm}$ length), chest radiograph showed cardiomegaly, serum ferritin was $171 \mathrm{ng} / \mathrm{ml}$, transferrin saturation was $21 \%$, echocardiogram showed concentric left ventricular hypertrophy, grade 2 left ventricular diastolic dysfunction, ejection fraction was $60 \%$.

On admission, he was initiated on hemodialysis after inserting double lumen hemodialysis catheter into right internal jugular vein. After few sessions of hemodialysis, he was subjected to right renal biopsy using real time ultrasound guided 16 gauge Bard automated renal biopsy gun after checking bleeding parameters like platelet count, prothrombin(PT) and activated partial thromboplastin time (aPTT) which were normal, serum creatinine was $2.16 \mathrm{mg} / \mathrm{dl}$, and blood urea was $59 \mathrm{mg} / \mathrm{dl}$. The procedure was uneventful and there was no immediate peri-nephric hematoma post-biopsy.

Renal biopsy showed chronic glomerulosclerosis. Light microscopy showed 9 Glomeruli, of which 8 were obsolescent. One viable glomerulus showed segmental sclerosis with synechiae. Capillary loops were patent with single basement membrane. No crescents, fibrinoid necrosis nor mesangial proliferation were noted. There was marked tubule-interstitial chronicity (approximately $>50 \%$ of cortical surface area) including thyroidization of tubules. Mild interstitial lymphocytic infiltrate was noted. Vessels showed intimal hyalinosis. No vasculitis or luminal thrombosis was noted.

Immunofluorescence was negative for antisera against complements and immunoglobins. Kappa and lambda stain showed no restriction.

72 hours later, he complained severe right loin pain following a heparin free hemodialysis. He was noted to be pale, hypotensive, but he did not have visible hematuria.

Ultrasound study showed a massive right perinephric hematoma with active ongoing bleed without extension into renal pelvis. Drop

*Corresponding author: Girish P Vakrani, A- 29, Vydehi Hospital Staff Quarters 82, EPIP Area, White Field, Bangalore-66, India, Tel: +91 9448988724; E-mail dr_vakranis@yahoo.co.in

Received July 18, 2013; Accepted September 17, 2013; Published September 21, 2013

Citation: Vakrani GP, Subramanyam NT, Babu H (2013) Perinephric Hematoma Following Renal Biopsy: A Case Report and Literature Review. J Nephrol Ther 3 139. doi:10.4172/2161-0959.1000139

Copyright: (c) 2013 Vakrani GP, et al. This is an open-access article distributed under the terms of the Creative Commons Attribution License, which permits unrestricted use, distribution, and reproduction in any medium, provided the original author and source are credited. 
Citation: Vakrani GP, Subramanyam NT, Babu H (2013) Perinephric Hematoma Following Renal Biopsy: A Case Report and Literature Review. J Nephrol Ther 3: 139. doi:10.4172/2161-0959.1000139

Page 2 of 3

in hemoglobin to $6 \mathrm{~g} / \mathrm{dl}$, Leucocytosis without neutrophilia, serum creatinine of $2.16 \mathrm{mg} / \mathrm{dl}$, blood urea of $31 \mathrm{mg} / \mathrm{dl}$, with normal PT, aPTT, platelet count were noted. Hemostatics, blood products, plasma expanders were transfused. Empirically he was covered with antibiotics.

Unfortunately he expired in very short time before definitive management like renal angiogram and intervention was attempted.

\section{Discussion}

The risks associated with performing a percutaneous renal biopsy have substantially decreased in the past two decades because of technical advances in the method. However, bleeding complications still occur, resulting in increased hospital stay, treatment costs and even mortality [1].

Independent predictors of postbiopsy bleeding are anaemia, raised baseline blood urea, serum creatinine, high diastolic blood pressure, need of hemodialysis. Needle size used for biopsy would not have impact on outcome. Bleeding occurred considerably later in duration after biopsy as noted in this study and may be triggered by hemodialysis though it was heparin free [2]. This finding could be related to the role of uremia in platelet dysfunction. An in vitro study showed that an increase in urea nitrogen altered the platelet aggregation process [3], and clinical studies have suggested that there is an increased risk of developing a hemorrhagic complication after PRB in patients with uremic syndrome $[3,4]$. Although other studies have demonstrated an association between serum creatinine levels and complications $[5,6]$.

The mechanism by which diastolic hypertension increases the development of major complications perhaps is related to the known association of diastolic hypertension and the development of microbleeds [2], which associated with renal puncture per se may increase the risk of developing major bleeding [7-9].

The biopsy technique has significantly improved over the past few decades because of the introduction of ultrasonography and automated-gun biopsy devices, and the incidence of life-threatening complications has come down significantly. The development of clinically apparent complications, such as gross hematuria, significant decrease in hemoglobin, hematomas, arteriovenous fistulas (AVFs), or severe flank pain post-biopsy has been reported to be between $7 \%$ and $15 \%[10,11]$

Severe loin pain immediately after renal biopsy in a patient with renal failure warrants careful follow-up of hemoglobin and imaging, even if initial imaging is normal. Further fall of hemoglobin necessitates early evaluation with angiogram, which helps in diagnosing the treatable, although rare, complications [10].

The incidence of clinically significant hematomas ranges between $2 \%$ and $3 \%$. Peri-renal bleeding usually occurs immediately after biopsy but can be delayed for some days or even weeks. Although clinically significant peri-nephric hematomas occur in $6 \%$ or fewer of biopsies, peri-nephric hematomas have been demonstrated at 24-72 hours after biopsy in $>90 \%$ of cases evaluated prospectively $[11,12]$.

Uremic patients have a bleeding tendency associated with platelet dysfunction, because platelets from uremic patients have a reduced aggregating response to adenosine diphosphate, epinephrine, and collagen. Uremic platelets also have a defective interaction with vessel subendothelium, and radioligand studies have indicated an impaired binding of fibrinogen to adenosine diphosphate-stimulated uremic platelets. Anemia, increases in nitric monoxide, and irregularities in von Willebrand factor are also related to a bleeding tendency, regardless of the platelet status [13]

Prevention and treatment options for bleeding include one or a combination of the following: erythropoietin, cryoprecipitate, desmopressin, and conjugated estrogens [13].

Several factors such as heparinization during dialysis, functional platelets abnormalities, intimal arterial fibrosis often combine to cause perirenal haemorrhage (Figure 1 and 2) [14].

Our patient developed peri-nephric hemorrhage despite having well-controlled blood pressure, not using anti-platelet agents, and normal pre-biopsy bleeding and coagulation parameters. The only risk factor the patient had at the time of renal biopsy was severe renal failure.

Severe loin pain that requires analgesia and sedation immediately after renal biopsy in a patient with renal failure warrants careful follow-up of hemoglobin and imaging, even if initial imaging is

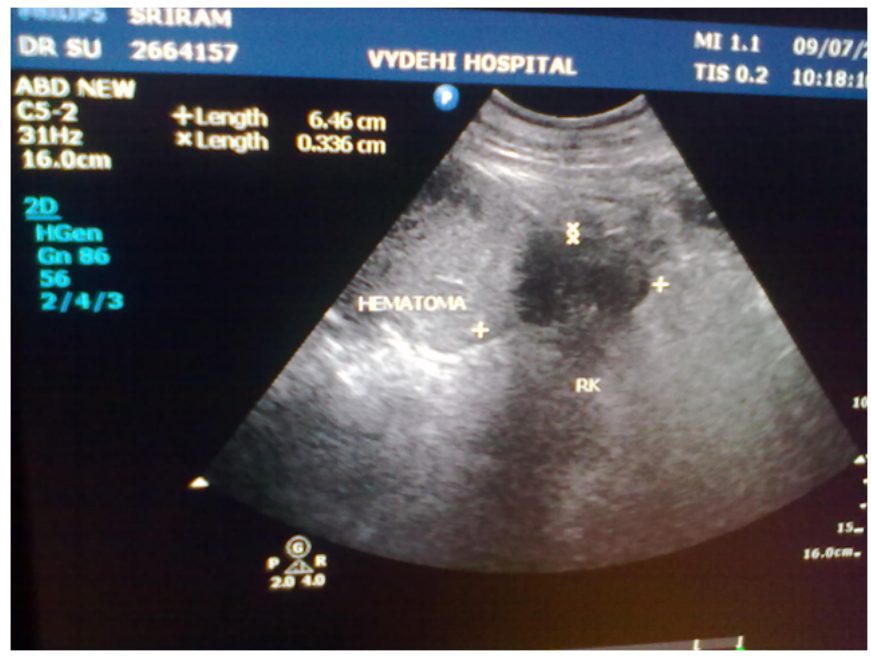

Figure 1: Huge perinephric hematoma compressing right kidney on ultrasound

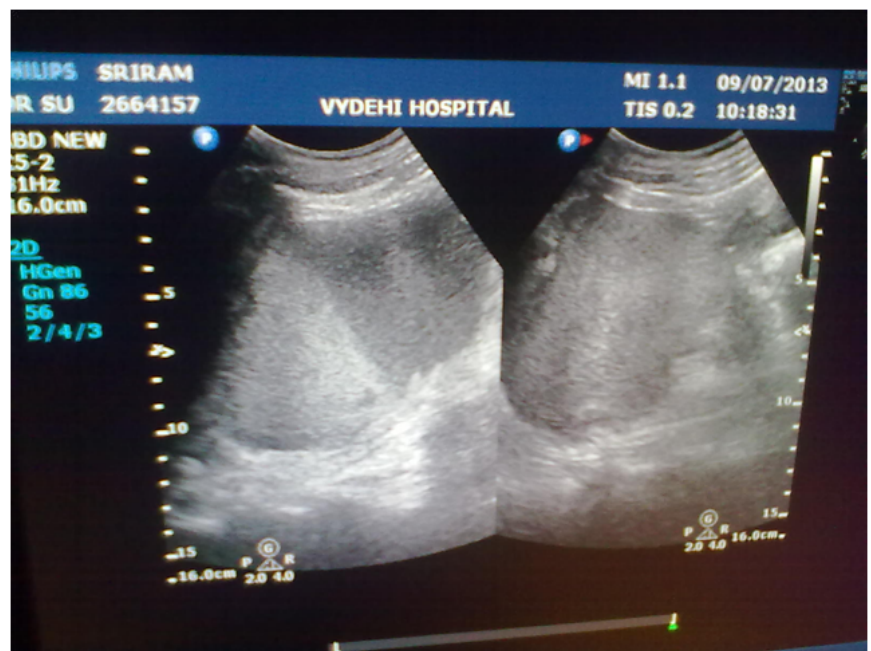

Figure 2: Huge perinephric hematoma compressing right and left kidney on ultrasound. 
Citation: Vakrani GP, Subramanyam NT, Babu H (2013) Perinephric Hematoma Following Renal Biopsy: A Case Report and Literature Review. J Nephrol Ther 3: 139. doi:10.4172/2161-0959.1000139

Page 3 of 3

negative. Further fall of hemoglobin necessitates early evaluation with angiogram, which helps in diagnosing as well as treating rare life threatening complications.

\section{Conclusion}

To the best of our knowledge, this is one of few reports of the occurrence of life threatening complication like perinephric hematoma in a patient with severe renal failure on heparin free hemodialysis 72 hours following a renal biopsy.

\section{References}

1. Manno C, Strippoli GF, Arnesano L, Bonifati C, Campobasso N, et al. (2004) Predictors of bleeding complications in percutaneous ultrasound-guided renal biopsy. Kidney Int 66: 1570-1577.

2. Whittier WL, Korbet SM (2004) Timing of complications in percutaneous renal biopsy. J Am SocNephrol 15: 142-147.

3. Steiner RW, Coggins C, Carvalho AC (1979) Bleeding time in uremia: a useful test to assess clinical bleeding. Am J Hematol 7: 107-117.

4. Shidham GB, Siddiqi N, Beres JA, Logan B, Nagaraja HN, et al. (2005) Clinical risk factors associated with bleeding after native kidney biopsy. Nephrology (Carlton) 10: 305-310.

5. (1988) Clinical competence in percutaneous renal biopsy. Health and Public Policy Committee. American College of Physicians. Ann Intern Med 108: $301-$ 303
6. Parrish AE (1992) Complications of percutaneous renal biopsy: a review of 37 years' experience. Clin Nephrol 38: 135-141.

7. Madaio MP (1990) Renal biopsy. Kidney Int 38: 529-543.

8. Corwin HL, Schwartz MM, Lewis EJ (1988) The importance of sample size in the interpretation of the renal biopsy. Am J Nephrol 8: 85-89.

9. Torres Muñoz A, Valdez-Ortiz R, González-Parra C, Espinoza-Dávila E Morales-Buenrostro LE, et al. (2011) Percutaneous renal biopsy of native kidneys: efficiency, safety and risk factors associated with major complications. Arch Med Sci 7: 823-831.

10. Madhav D, Ram R, Rammurti S, Dakshinamurty KV (2011) Severe loin pain following renal biopsy in a high-risk patient: a case report of a rare combination of pseudoaneurysm and arterio-venous fistula. Saudi J Kidney Dis Transpl 22: 544-548.

11. Ivan D Maya, Michael Allon, Souheil Saddekni (2008) Interventional Nephrology. Brenner and Rector's The Kidney (8thedn) 1: 915-941.

12. Rosenbaum R, Hoffsten PE, Stanley RJ, Klahr S (1978) Use of computerized tomography to diagnose complications of percutaneous renal biopsy. Kidney Int 14: 87-92.

13. Kawahara T, Kawahara K, Ito H, Yamaguchi S, Mitsuhashi H, et al. (2011) Spontaneous renal hemorrhage in hemodialysis patients. Case Rep Nephrol Urol 1: 1-6.

14. JaHyeon Ku, Jung-Kwon Kim, Seungbeom Ha, Lee JW (2009) Bilatera spontaneous perirenalhaemorrhage in a patient on haemodialysis. Clinical Kidney Journal 5: 412-414. 